\title{
Ginsenoside Rh1 Improves the Effect of Dexamethasone on Autoantibodies Production and Lymphoproliferation in MRL/lpr Mice
}

\author{
Yinglu Feng, ${ }^{1}$ Chunbin Wang, ${ }^{1}$ Silu Cheng, ${ }^{2,3}$ Xiaorong Wang, ${ }^{2,4}$ Xianze Meng, ${ }^{1}$ Lujia Li, \\ Juan Du, ${ }^{2}$ Qun Liu, ${ }^{2}$ Yuyu Guo, ${ }^{2}$ Yongbin Meng, ${ }^{2}$ Binbin Cheng, ${ }^{2}$ and Changquan Ling ${ }^{2,5}$ \\ ${ }^{1}$ Department of Traditional Chinese Medicine, 401 Hospital of the Chinese People's Liberation Army, Qingdao, Shandong 266071, China \\ ${ }^{2}$ Department of Traditional Chinese Medicine, Changhai Hospital, Second Military Medical University, Shanghai 200433, China \\ ${ }^{3}$ Department of Oncology, 534 Hospital of the Chinese People's Liberation Army, Luoyang, Henan 471003, China \\ ${ }^{4}$ Department of Oncology, Suzhou Hospital of Traditional Chinese Medicine, Suzhou, Jiangsu 215009, China \\ ${ }^{5}$ E-Institute of TCM Internal Medicine, Shanghai Municipal Education Commission, Shanghai 201203, China
}

Correspondence should be addressed to Changquan Ling; lingchangquan@hotmail.com

Received 10 December 2014; Accepted 11 March 2015

Academic Editor: Fabio Firenzuoli

Copyright (C) 2015 Yinglu Feng et al. This is an open access article distributed under the Creative Commons Attribution License, which permits unrestricted use, distribution, and reproduction in any medium, provided the original work is properly cited.

\begin{abstract}
Ginsenoside Rh1 is able to upregulate glucocorticoid receptor (GR) level, suggesting Rh1 may improve glucocorticoid efficacy in hormone-dependent diseases. Therefore, we investigated whether Rhl could enhance the effect of dexamethasone (Dex) in the treatment of MRL/lpr mice. MRL/lpr mice were treated with vehicle, Dex, Rh1, or Dex + Rh1 for 4 weeks. Dex significantly reduced the proteinuria and anti-dsDNA and anti-ANA autoantibodies. The levels of proteinuria and anti-dsDNA and anti-ANA autoantibodies were further decreased in Dex + Rh1 group. Dex, Rh1, or Dex + Rh1 did not alter the proportion of CD4+ splenic lymphocytes, whereas the proportion of CD8+ splenic lymphocytes was significantly increased in Dex and Dex + Rh1 groups. Dex + Rh1 significantly decreased the ratio of CD4+/CD8+ splenic lymphocytes compared with control. Con A-induced CD4+ splenic lymphocytes proliferation was increased in Dex-treated mice and was inhibited in Dex + Rhl-treated mice. Thl cytokine IFN- $\gamma$ mRNA was suppressed and Th2 cytokine IL- 4 mRNA was increased by Dex. The effect of Dex on IFN- $\gamma$ and IL- 4 mRNA was enhanced by Rh1. In conclusion, our data suggest that Rh1 may enhance the effect of Dex in the treatment of MRL/lpr mice through regulating CD4+ T cells activation and Th1/Th2 balance.
\end{abstract}

\section{Introduction}

Systemic lupus erythematosus (SLE), a chronic autoimmune disease, is characterized by the presence of autoantibodies and the deposition of immune complexes in multiple organs, including the skin, kidneys, heart, and joints, resulting in inflammation and tissue damage $[1,2]$. To date, glucocorticoids (GCs) are still the first-line drugs for SLE. Although most patients with active SLE benefit from the anti-inflammatory action of GCs, approximately $30 \%$ of SLE patients do not respond sufficiently and thus require a higher dose [3]. However, high dose and/or prolonged GCs administration usually causes severe side effects, such as osteoporosis, cardiovascular events, diabetes mellitus, infection, and hemorrhage of digestive tract that seriously affect the quality of life and the prognosis of patients [4].

The reasons for GC insensitivity have been widely reported previously, such as decreased expression of glucocorticoid receptor alpha $(\mathrm{GR} \alpha)$, which is a member of the nuclear receptor family of ligand-dependent transcription factors and mediates most GCs actions, reduced DNAbinding ability of GR $\alpha$, increased GRbeta $(\beta)$ transcript levels, and enhanced expression of inflammatory transcription factors like AP-1 or NF $\kappa \mathrm{B}$ [5-8]. It is widely recognized that 
the level of GR $\alpha$ expression is closely correlated with GCs response [9]. However, administration of GR $\alpha$ agonists usually downregulates GR $\alpha$ levels and thereby limits the therapeutic responses to GCs. In contrast, upregulation of GR $\alpha$ is associated with enhanced glucocorticoid sensitivity. Therefore, agents that may upregulate the expression of GR $\alpha$ in the process of GC treatment would enhance the efficacy of GCs in SLE patients and thereby reduce the GCs dose and side effects.

In the past decade, our group continuously focused on the regulation of GR with Traditional Chinese Medicine [10, 11]. Ginsenosides (GSS), the main extracts of ginseng, are able to partially reverse dexamethasone (Dex) induced downregulation of GR expression and hormone binding activity in HL7702 cells and subsequently enhance Dex induced transcription of reporter gene [12]. This effect was also confirmed in rats [10]. Among the numerous ingredients of GSS, Rh1 has been identified as an effective part accounting for the GR upregulation after Dex treatment and enhances the antiinflammatory effect of Dex in collagen-induced arthritis (CIA) mouse model [13]. This exciting result suggests Rh1 may be also effective in improving GCs efficacy in other hormone-dependent diseases. Therefore, we further investigated the effect of Rh1 in combination with Dex in the treatment of SLE mouse model.

\section{Materials and Methods}

2.1. Animals. Eight-week old female MRL/lpr mice were purchased from Shanghai Laboratory Animal Center and maintained in standard animal cages under specific pathogen-free conditions in the Laboratory Animal Center of the Second Military Medical University with the dark/light cycle of $12 \mathrm{~h}$ at $22^{\circ} \mathrm{C}$. The animals were maintained with food and water available ad libitum and housed for a week prior to the experiment. The experiments were performed in accordance with the European Communities Council Directive of 24 November 1986 (86/609 EEC) and approved by the Ethics Committee of Changhai Hospital.

2.2. Drug Administration. Twenty-four female MRL/lpr mice were randomly divided into 4 groups: (1) control group; (2) Dex group; (3) Rh1 group; (4) Dex + Rhl group. Mice were intraperitoneally injected with $10 \%$ ethanol (vehicle for Rh1 and Dex), Dex (1 mg. $\left.\mathrm{kg}^{-1} \cdot \mathrm{d}^{-1}\right)$, Rh1 (25 mg. $\left.\mathrm{kg}^{-1} \cdot \mathrm{d}^{-1}\right)$, or Dex + Rh1 daily for 4 weeks. All the animals were killed by decapitation $12 \mathrm{~h}$ after the last injection. Approximately $0.8 \mathrm{~mL}$ trunk blood was collected into centrifuge tubes containing $100 \mu \mathrm{L} 0.3 \mathrm{M}$ EDTA for radioimmunoassay. Collected blood was immediately centrifuged by using a Sorvall RC-3 centrifuge at $3000 \mathrm{~g}$ for $15 \mathrm{~min}$. The supernatant was harvested and stored at $-70^{\circ} \mathrm{C}$ for future use.

2.3. The Weight Ratio of the Spleen in Mice. The weight ratio of spleen is calculated according to the following formula: weight of an organ $(\mathrm{mg}) /$ body weight of a mouse $(\mathrm{g}) \times 100 \%$.

2.4. Measurement of Antibodies. Serum levels of antibodies reactive to double stranded DNA (dsDNA), antinuclear antibody (ANA), and antimitochondrial antibody (AMA) were measured by ELISA, as described previously [14].

2.5. Assessment of Proteinuria. To test the urinary protein excretion, mice were placed in metabolic cages after the last administration and urine samples were collected over a $24 \mathrm{~h}$ period. Protein concentrations were determined using urine protein test kit (Nanjing Jiancheng Bioengineering Institute, Nanjing, China), according to the manufacturer's instructions.

2.6. Isolation of Splenic T Cells. Mouse spleens were harvested and washed twice with ice-cold phosphate-buffered saline (PBS). Spleens were grinded in cold PBS containing 2\% FBS and filtered with a 200 mesh strainer. Cells were washed in PBS containing 2\% FBS by centrifugation at $1200 \mathrm{rpm}$ at $4^{\circ} \mathrm{C}$ for $10 \mathrm{~min}$ and the supernatants were discarded. After resuspension in $1 \mathrm{~mL}$ PBS containing $2 \%$ FBS, $100 \mu \mathrm{L}$ aliquots of cell suspension were added to the appropriate tubes. Monoclonal antibodies (CD3-FITC, CD8-PerCP, and CD4-PE, $10 \mu \mathrm{L}$ for each) were added along with appropriate isotypic negative. These tubes were incubated for $10 \mathrm{~min}$ at $4^{\circ} \mathrm{C}$. Then $0.5 \mathrm{~mL}$ Red Blood Cell Lysis Buffer (R\&D systems, Minneapolis, MN, USA) was added in the tubes and the cells were centrifuged at $1200 \mathrm{rpm}$ for $10 \mathrm{~min}$. The cells were washed with $1 \mathrm{~mL}$ PBS, centrifuged at rpm for $10 \mathrm{~min}$, and resuspended in $0.2 \mathrm{~mL}$ PBS. For each sample, 10000 events were analyzed and the proportions of CD3+CD4+ and $\mathrm{CD} 3+\mathrm{CD} 8+$ splenic lymphocytes were determined by flow cytometry and CD3+CD4+ T cells were isolated for the next experiments. The ratio of CD $4+/ C D 8+T$ cells was then calculated.

2.7. T Cell Proliferation Assay. CD3+CD4+ T cells were isolated by flow cytometry and then suspended in RPMI 1640 culture medium and placed in 96-well round-bottom plates at a cell density of $2 \times 10^{6} / \mathrm{mL}$ to proliferate. RPMI-1640 medium with or without concanavalin A (Con A, $5 \mathrm{mg} / \mathrm{L}$, Sigma, St. Louis, Mo, USA) was added and incubated at $37^{\circ} \mathrm{C}$ under $5 \% \mathrm{CO}_{2}$ for $72 \mathrm{~h}$. Then, $10 \mu \mathrm{L}$ 3-(4,5-dimethyl-2-thiazolyl)2,5-diphenyl-2-H-tetrazolium bromide (MTT, $5 \mathrm{mg} / \mathrm{mL}$ ) was added to each well, and then plate was incubated for another $4 \mathrm{~h}$. Finally, $100 \mu \mathrm{L}$ SDS-isobutanol-HCl solution (10\% SDS, $5 \%$ isobutanol, and $12 \mathrm{Mm} \mathrm{HCl}$ ) was added to dissolve the MTT crystals $[15,16]$. The optical density was measured with a microplate reader at the wavelength of $570 \mathrm{~nm}$.

2.8. Real-Time RT-PCR Analysis of Cytokine mRNA in CD4+ Spleen Cells. Real-time RT-PCR was performed as described previously $[17,18]$. Primers used for each cytokine are listed as follows: IFN- $\gamma$, forward: $5^{\prime}$-GTC AAC AAC CCA CAG GTC CA- $3^{\prime}$; reverse: $5^{\prime}$-CGA CTC CTT TTC CGC TTC CT-3'; IL-4, forward: $5^{\prime}$-ATG GAT GTG CCA AAC GTC CT- $3^{\prime}$; reverse: $5^{\prime}-\mathrm{AAG}$ CAC CTT GGA AGC CCT AC-3'; $\beta$-actin, forward: $5^{\prime}$-CCT CTA TGC CAA CAC AGT GC$3^{\prime}$; reverse: $5^{\prime}$-GTA CTC CTG CTT GCT GAT CC-3'. The relative expression of $\mathrm{mRNA}$ in each sample was normalized to its $\beta$-actin. The relative expression of mRNA was calculated as $2^{-\Delta \Delta \mathrm{Ct}}$. 


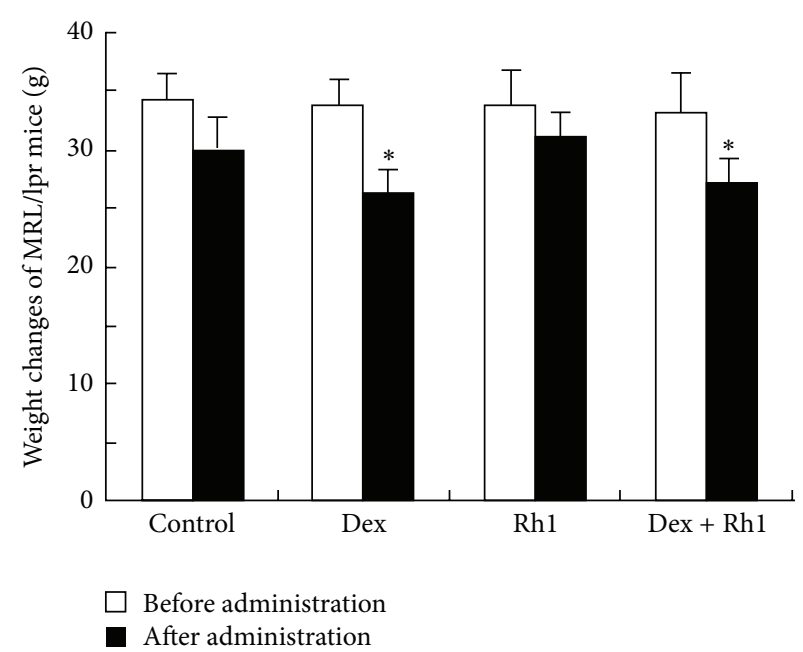

(a)

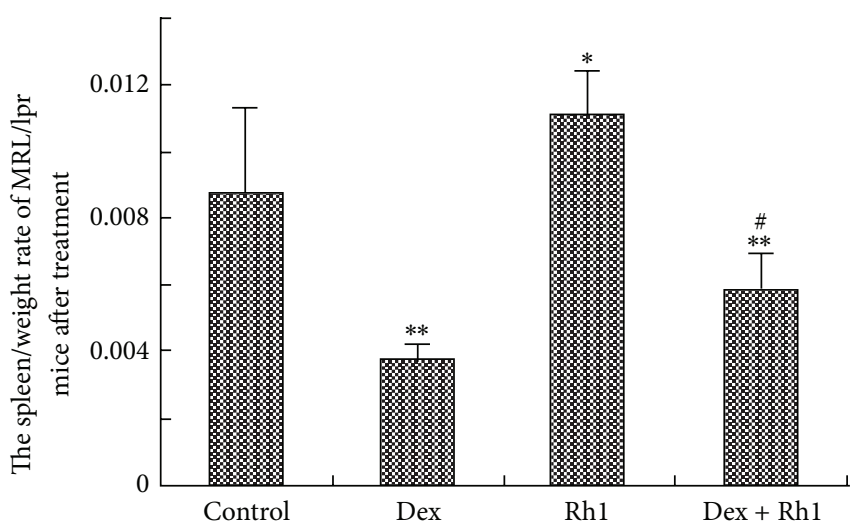

(b)

FIGURE 1: The effect of Dex in combination with Rhl on the body weight and spleen/weight ratio of MRL/lpr mice. (a) The body weight changes MRL/lpr mice before and after treatment; (b) the spleen/weight ratio of MRL/lpr mice after 4-week treatment. Each bar represents mean \pm S.D. $(n=6) .{ }^{*} P<0.05,{ }^{* *} P<0.01$, compared with control; ${ }^{\#} P<0.05$, compared with Dex group.

2.9. Statistical Analysis. All data were presented as the means \pm standard deviation (S.D). Statistical significance was determined using SPSS 18.0 for Windows. Data analysis was performed by one-way analysis of variance (ANOVA), followed by Fisher's LSD. Differences with $P$ values $<0.05$ were considered to be statistically significant.

\section{Results}

3.1. Rh1 Increased the Spleen/Weight Ratio of MRL/lpr Mice. Compared with MRL/lpr mice, the body weights of Dexand Dex + Rh1-treated mice were significantly decreased (Figure 1(a)). Rh1 alone showed little effect on the mice body weight compared with control group. There were no significant differences in the body weight between Dex and Dex + Rh1 groups (Figure 1(a)). The spleen/weight ratio in Dex group was significantly decreased compared with that in control group (Figure 1(b)). In the Rhl group, the spleen/weight ratio was significantly increased compared with control group. The spleen/weight ratio of Dex + Rh1treated mice was lower than that of MRL/lpr mice, but higher than that of Dex-treated mice.

\subsection{Rh1 Enhanced the Effect of Dex on the Levels of Proteinuria,} Anti-dsDNA, and Anti-ANA Antibodies in MRL/lpr Mice. To determine the therapeutic effect of Dex, Rh1, or Dex + Rh1 on MRL/lpr mice, we evaluated whether treatment with Dex, Rh1, or Dex + Rh1 improved the proteinuria and autoantibody production. Both Dex and Rhl treatment significantly reduced the proteinuria levels (Figure 2(a)). The proteinuria level in Dex + Rhl group was further decreased compared with that in Dex group. Compared with MRL/lpr mice, the levels of anti-dsDNA and anti-ANA autoantibodies in Dextreated mice were significantly decreased (Figures 2(b) and 2(c)). In the Dex + Rhl group, the levels of anti-dsDNA and anti-ANA autoantibodies were further downregulated compared with those of Dex group. Although the antidsDNA and anti-ANA levels were lower in Rhl group than in control group, the differences between the two groups were not significant.

3.3. Proportion of CD3+CD4+ and CD3+CD8+ Splenic Lymphocytes. $\mathrm{CD} 3+\mathrm{CD} 4+\mathrm{T}$ cells are required for the development of human LE-like disease, and anti-CD4 antibody therapy decreases lymphoproliferation and autoantibody production and protects renal functions in MRL/lpr mice [19-21]. Therefore, we further examined the effect of Rhl in combination with Dex on CD4+ and CD8+ splenic lymphocytes. After 4-week treatment, the proportion of CD4+ splenic lymphocytes was not significantly altered by Dex, Rh1, or Dex + Rh1 compared with control (Figure 3(b)). Meanwhile, the proportion of CD8+ splenic lymphocytes was significantly increased in Dex and Dex + Rhl groups compared with control (Figure 3(b)). But there was no significant difference between Dex group and Dex + Rh1 group. As shown in Figure 3(c), the ratio of $\mathrm{CD} 4+/ \mathrm{CD} 8+$ splenic lymphocytes was $2.12 \pm 0.34$ in control group, $1.38 \pm 0.53$ in Dex group, $2.43 \pm 0.69$ in Rhl group, and $0.96 \pm 0.06$ in Dex + Rhl group. There was a significant difference between control group and Dex + Rh1 group. However, no significant difference was determined between Dex group and control group and between Dex group and Dex + Rhl group.

3.4. Dex Increased Con A-Induced Proliferation of CD4+ Splenic Lymphocytes. As shown by previous study [22], CD4+ splenic lymphocytes isolated from control mice spleen displayed a deficient proliferative response to Con A (Figure 4). Dex treatment increased splenic lymphocytes proliferation in response to various concentrations of Con A. However, the proliferation of splenic lymphocytes in response to various 


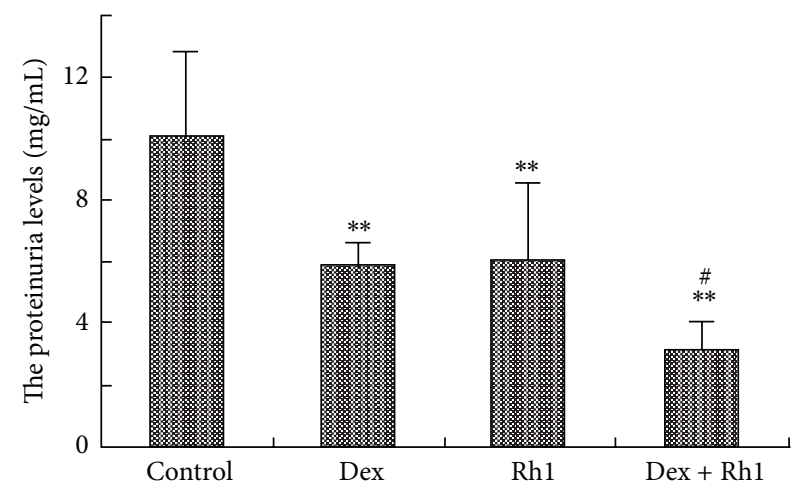

(a)

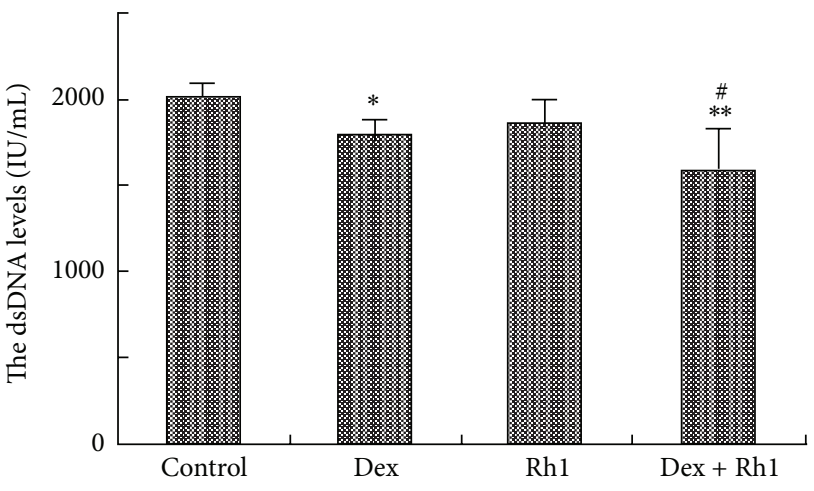

(b)

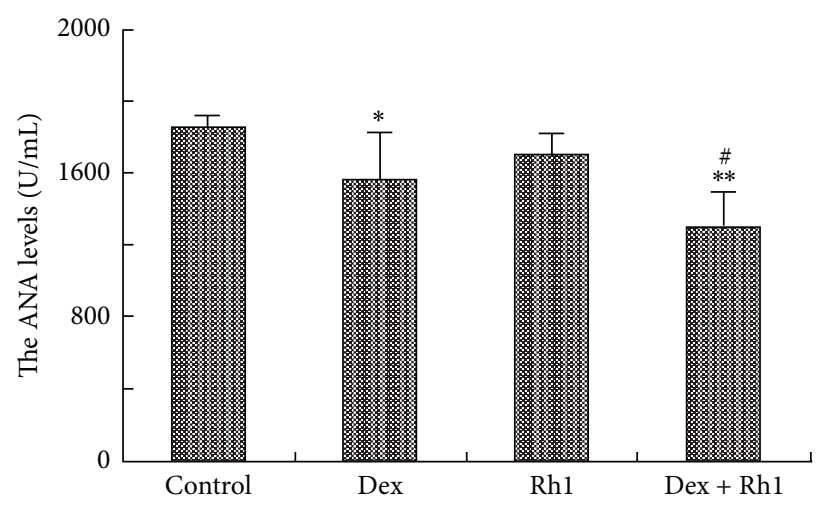

(c)

FIgURE 2: Rhl improved the effect of Dex on proteinuria and anti-dsDNA and anti-ANA antibodies of MRL/lpr mice. (a) The proteinuria levels of MRL/lpr mice after treatment; (b) the anti-dsDNA levels of MRL/lpr mice after treatment; (c) the anti-ANA levels of MRL/lpr mice after treatment. Each bar represents mean \pm S.D. $(n=6) .{ }^{*} P<0.05,{ }^{* *} P<0.01$, compared with control; ${ }^{*} P<0.05$, compared with Dex group.

concentrations of Con A was decreased in Rh1 + Dex group compared with Dex group.

3.5. Expression of Th1/Th2 Cytokine mRNA in CD4+ Splenic Lymphocytes. IFN- $\gamma$ and IL-4 are major mediators of several autoimmune and inflammatory diseases. Therefore, we determined the mRNA levels of IFN- $\gamma$ and IL-4 in CD4+ splenic lymphocytes from MRL/lpr mice that were treated with Dex, Rh1, or Dex + Rh1. As shown in Figure 5(a), the expression of IFN- $\gamma$ mRNA was suppressed by Dex and Rhl compared with control. In the Dex + Rhl group, the IFN- $\gamma$ mRNA level was further decreased compared with Dex group. In contrast, the IL-4 mRNA was increased by Dex and Rhl compared with control (Figure 5(b)). Rh1 in combination with Dex induced a further increase of IL-4 mRNA level compared with Dex alone.

\section{Discussion}

MRL/lpr mice develop a systemic autoimmune disease similar to SLE in humans. Although GCs and several immunosuppressive agents can effectively control the disease activities, patients may also suffer from serious adverse effects caused by the treatment agents. In the present study, our data demonstrated that Rhl is able to enhance the ability of Dex on the reduction of proteinuria and autoantibodies in MRL/lpr mice. After 4-week treatment, Dex combined with Rh1 increased the proportion of CD8+ splenic lymphocytes and decreased the ratio of CD4+/CD8+ splenic lymphocytes, whereas it showed little effect on the proportion of CD4+ splenic lymphocytes. Con A-induced proliferation of CD4+ splenic lymphocytes was increased by Dex, whereas it is suppressed by Rhl cotreatment. Rhl also enhanced the ability of Dex on the reduction of IFN- $\gamma$ mRNA and the increase of IL-4 mRNA.

It has been reported that elevated GCs levels induce spleen lymphocyte apoptosis, which may account for lymphocytes reduction and spleen atrophy [23]. GCs evoke apoptosis through regulating $\mathrm{Bax}$ and $\mathrm{Bcl}-2$ mRNA and proteins expression after activating glucocorticoids receptor (GR) [24]. Our results showed that Dex treatment markedly decreased the spleen spleen/weight ratio, whereas spleen/weight ratio of Dex + Rhl-treated mice was higher than that of Dex-treated mice, suggesting that cotreatment with Rhl could prevent the reduction of spleen lymphocyte number and spleen atrophy induced by Dex. 

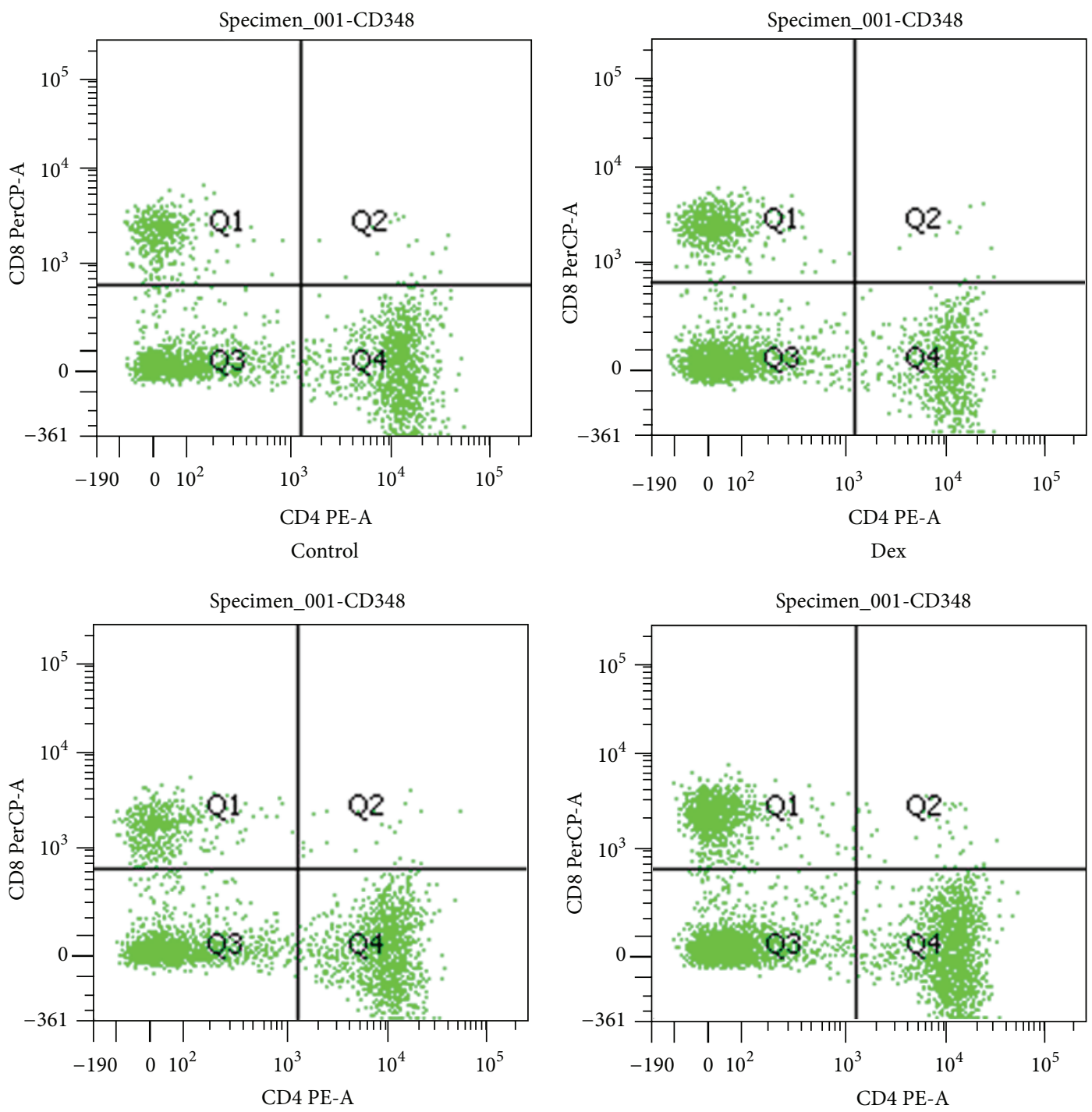

Rh1

$\mathrm{Dex}+\mathrm{Rh} 1$

(a)

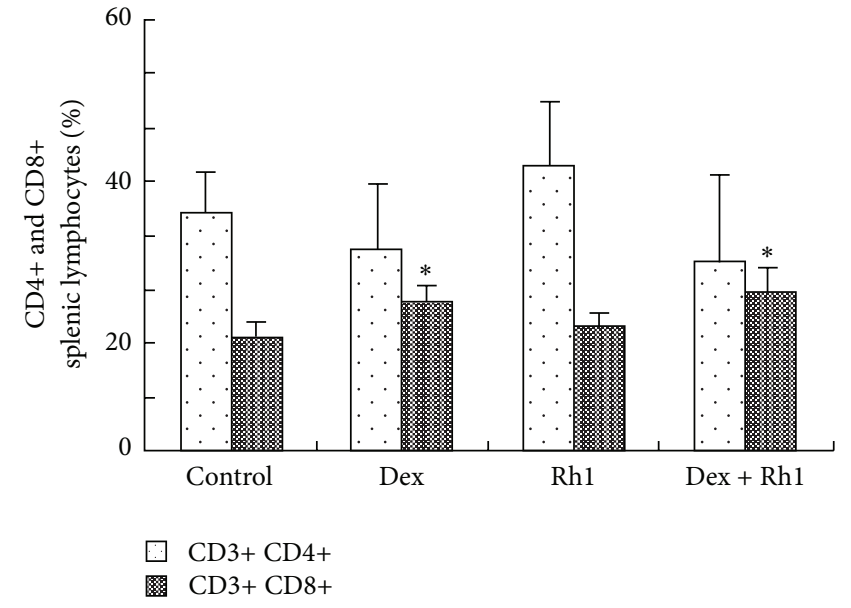

(b)

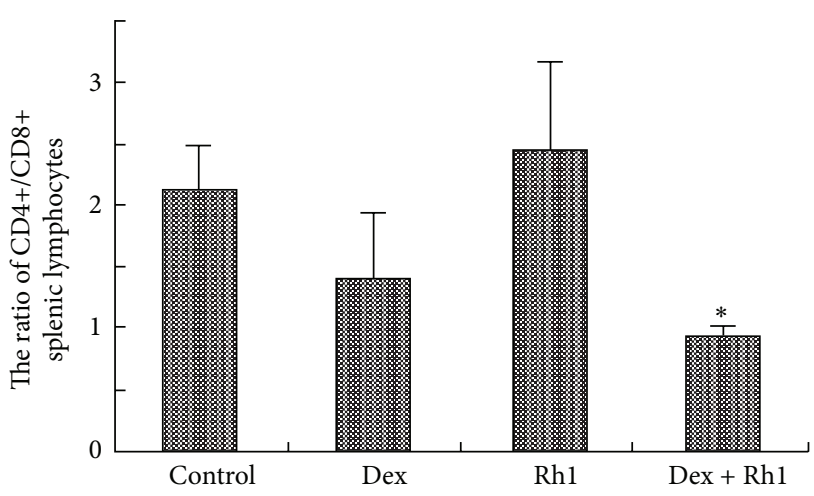

(c)

FIGURE 3: The proportion of CD3+CD4+ and CD3+CD8+ splenic lymphocytes after treatment. (a) Analysis of CD3+CD4+ and CD3+CD8+ splenic lymphocytes. Splenic lymphocytes were obtained from 3 mice randomly selected from each group. Splenic lymphocytes were stained by monoclonal antibodies against CD3, CD8, and CD4 and then analyzed by flow cytometry; (b) the proportion of CD3+CD4+ and $\mathrm{CD} 3+\mathrm{CD} 8+$ splenic lymphocytes; (c) the ratio of CD4+/CD8+ splenic lymphocytes. Each bar represents mean \pm S.D. $(n=3) .{ }^{*} P<0.05$, compared with control. 


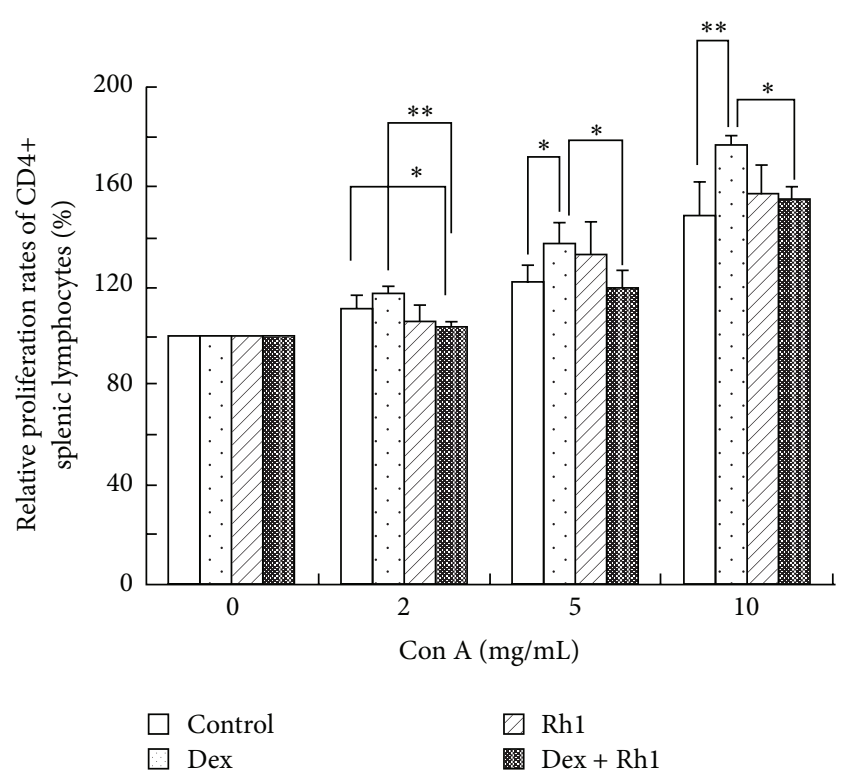

FIGURE 4: Proliferation of CD4+ splenic lymphocytes. CD4+ splenic lymphocytes isolated from mice spleen were stimulated with indicated concentrations of Con A for $72 \mathrm{~h}$. MTT assay was performed to determine the proliferation of cells. Each bar represents mean \pm S.D. $(n=3) .{ }^{*} P<0.05,{ }^{* *} P<0.01$.

In clinical practice, an insufficient response of an SLE patient to a given dose of GCs (GC-resistance) leads to prescription of a higher dose ( $>30 \mathrm{mg} /$ day) or additional immunosuppressive drugs. Furthermore, it was reported that approximately $30 \%$ of patients failed to respond to even high doses of glucocorticoids $[1,2]$. Serious side effects will also present in those patients who take great a dosage of GC for long time. The downregulation of GC on GR is one of the reasons of GC resistance and could therefore seriously decrease the efficacy of GC used clinically [13]. Therefore, it is critical to the development of newer more effective therapies for these difficult to manage disease conditions.

SLE is mainly characterized by the abnormal increase of autoantibodies [25]. The anti-dsDNA is usually used as a biomarker for the diagnosis of SLE and evaluation of its activity in patients [26-28]. The presence of ANA, found in 95\% of SLE patients, is also a prevalent feature of SLE that plays a pivotal role in the disease pathogenesis [29]. The level of ANA is positively correlated with anti-dsDNA [30,31]. As expected, Dex-treated MRL/lpr mice showed a remarkable decrease in anti-dsDNA and anti-ANA autoantibodies. The effect of Dex on autoantibodies was strengthened by Rhl. However, $\mathrm{Rh} 1$ alone showed little effect on these autoantibodies. Lupus nephritis is one of the most severe symptoms related to the cause of death in human SLE. Our study also demonstrated that the level of proteinuria was further decreased by Dex combined with Rh1 compared with Dex alone. Therefore, our data suggest that Rhl may be also able to potentiate the effect of Dex in the treatment of SLE.

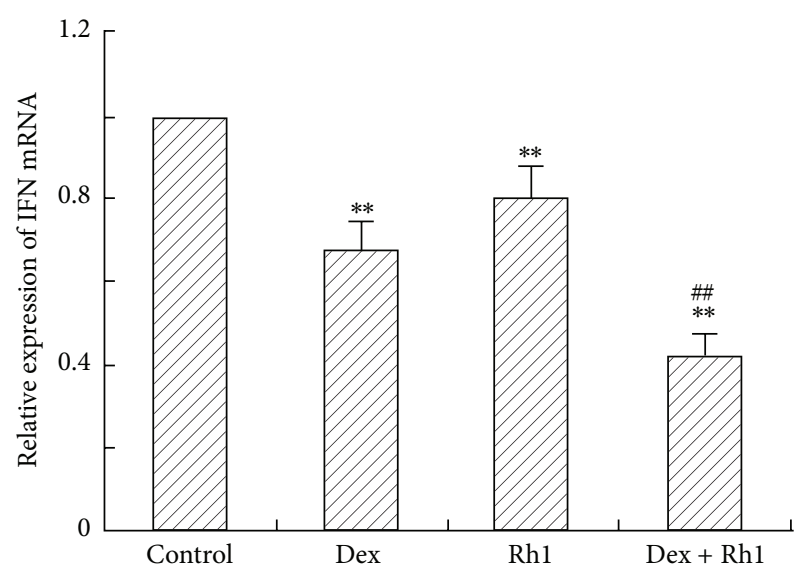

(a)

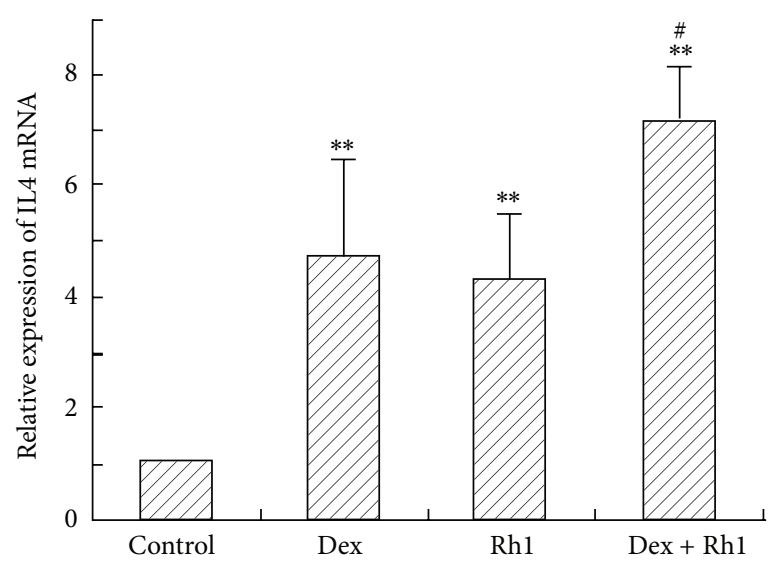

(b)

FIGURE 5: Expression of IFN- $\gamma$ and IL-4 mRNA in the CD4+ splenic lymphocytes. Total RNA was isolated from CD4+ splenic lymphocytes and then real-time RT-PCR was performed to determine the level of IFN- $\gamma$ and IL- 4 mRNA. The expression of IFN- $\gamma$ and IL-4 mRNA was normalized by $\beta$-actin. Each bar represents mean \pm S.D. $(n=3) .{ }^{* *} P<0.01$, compared with control; ${ }^{\#} P<0.05,{ }^{\#} P<0.01$, compared with Dex group.

Numerous studies indicate that the induction of antidsDNA autoantibodies in SLE are antigen-driven and thus $\mathrm{T}$ cell dependent response [32]. Koh et al. [33] showed that the disease was alleviated with a reduction in autoantibody production in CD4-/- lpr mice compared to littermate controls. The disease was also improved by $\mathrm{T}$ cell depletion and by combined anti-CD 4 and anti-CD $8 \mathrm{mAb}$ therapy but not by anti-CD $8 \mathrm{mAb}$ therapy alone [34], indicating that SLE is dependent on CD4+ T cells. In the present study, our data showed that both Dex and Rh1 did not change the proportion of $\mathrm{CD} 4+\mathrm{T}$ cells in spleen, whereas they increased the proportion of CD8+ T cells. Furthermore, Dex in combination with $\mathrm{Rh} 1$ decreased the ratio of $\mathrm{CD} 4+/ \mathrm{CD} 8+$ splenic lymphocytes. Moreover, the proliferation of CD4+ splenic lymphocytes isolated from Dex-treated MRL/lpr mice after Con A stimulation was speedy compared with the cells obtained from control MRL/lpr mice spleen. However, Rh1 cotreatment with Dex suppressed the proliferation of CD4+ 
splenic lymphocytes. Our data reveal that Rhl may inhibit the activation of CD4+ cells induced by Dex treatment.

Disturbed expression of Th1/Th2 cytokines is suggested to contribute to the pathogenesis of SLE $[35,36]$. Th1- and Th2-cells are able to express their key cytokines IFN- $\gamma$ and IL-4, respectively. In the peripheral blood mononuclear cells (PBMC) from SLE patients, the expression of IFN- $\gamma$ and IL10 mRNA is increased and IL-4 mRNA is decreased [37]. Sugimoto et al. [38] showed that the number of CD4+ T cells producing IFN $-\gamma$ was increased, and the number of CD4+ $\mathrm{T}$ cells producing IL-4 was significantly decreased in SLE patients. In the current study, our study showed that the expression of Th1 cytokine IFN- $\gamma$ mRNA was reduced in Dextreated mice. However, the IL-4 mRNA level was increased in the CD4+ splenic lymphocytes isolated from Dex-treated MRL/lpr mice. The effect of Dex on IFN- $\gamma$ and IL- 4 mRNA expression was further enhanced by the cotreatment of Rh1. It is widely recognized that GCs are able to inhibit the expression of IFN- $\gamma$ in MRL/lpr mice and SLE patients. However, the results about GCs on IL- 4 production by T cells are inconsistent $[39,40]$. GCs were reported to increase the production of IL- 4 by mouse T cells, whereas they suppressed the production of IL-4 by human lymphocytes [39, 40]. GCs also inhibit the serum levels of IL-4 and the mRNA expression in human peripheral blood CD4+ T cells [41, 42]. However, pretreatment with Dex increased IL-2 induced IL4 production in rat $\mathrm{CD} 4+\mathrm{T}$ cells, whereas it decreased the synthesis of IFN- $\gamma$ [43]. Dex decreased IFN- $\gamma$ production and increased IL-4 and IL-10 production by tetanus-stimulated human PBMC [44]. These diverse results may be due to the differences in cell types, species, or the treatment methods. In our study, we showed that Dex inhibited IFN- $\gamma$ production, while increasing IL-4 level, indicating GCs may induce a shift from the Th1 to Th2 profile of cytokine secretion, which may contribute to the mechanisms for GCs treatment.

Taken together, our study demonstrated that Rh1 may enhance the effect of Dex in the treatment of experimental SLE mice. Rh1 combined with Dex decreased the ratio of CD4+/CD8+ splenic lymphocytes but did not alter the proportion of CD4+ splenic lymphocytes. Rh1 combined with Dex also suppressed Con A-induced proliferation of CD4+ splenic lymphocytes and regulated the expression of Th1/Th2 cytokines. These results suggest that regulating CD4+ T cells activation and the ratio of Th1/Th2 cytokines may contribute to the enhancement of Dex on MRL/lpr mice. However, whether the Rh1 could also exhibit similar effect in humans needs to be further studied.

\section{Conflict of Interests}

The authors declare that there is no conflict of interests.

\section{Acknowledgment}

This study was supported by the grants from the National Natural Science Foundation of China (nos. 81173388 and 81202973).

\section{References}

[1] T. N. Marion and A. E. Postlethwaite, "Chance, genetics, and the heterogeneity of disease and pathogenesis in systemic lupus erythematosus," Seminars in Immunopathology, vol. 36, no. 5, pp. 495-517, 2014.

[2] V. Oke and M. Wahren-Herlenius, "Cutaneous lupus erythematosus: clinical aspects and molecular pathogenesis," Journal of Internal Medicine, vol. 273, no. 6, pp. 544-554, 2013.

[3] R. K. M. A. C. Luijten, R. D. Fritsch-Stork, J. W. J. Bijlsma, and R. H. W. M. Derksen, "The use of glucocorticoids in systemic lupus erythematosus. After 60 years still more an art than science," Autoimmunity Reviews, vol. 12, no. 5, pp. 617-628, 2013.

[4] J. Du, B. Cheng, X. Zhu, and C. Ling, "Ginsenoside Rgl, a novel glucocorticoid receptor agonist of plant origin, maintains glucocorticoid efficacy with reduced side effects," The Journal of Immunology, vol. 187, no. 2, pp. 942-950, 2011.

[5] A. Geiss, N. Rohleder, and F. Anton, "Evidence for an association between an enhanced reactivity of interleukin-6 levels and reduced glucocorticoid sensitivity in patients with fibromyalgia," Psychoneuroendocrinology, vol. 37, no. 5, pp. 671-684, 2012.

[6] J. A. Macedo, J. Hesse, J. D. Turner, J. Meyer, D. H. Hellhammer, and C. P. Muller, "Glucocorticoid sensitivity in fibromyalgia patients: decreased expression of corticosteroid receptors and glucocorticoid-induced leucine zipper," Psychoneuroendocrinology, vol. 33, no. 6, pp. 799-809, 2008.

[7] W. J. E. Tissing, J. P. P. Meijerink, M. L. den Boer, and R. Pieters, "Molecular determinants of glucocorticoid sensitivity and resistance in acute lymphoblastic leukemia," Leukemia, vol. 17, no. 1, pp. 17-25, 2003.

[8] J. Du, M. Li, D. Zhang et al., "Flow cytometry analysis of glucocorticoid receptor expression and binding in steroid-sensitive and steroid-resistant patients with systemic lupus erythematosus," Arthritis Research and Therapy, vol. 11, no. 4, article R108, 2009.

[9] J. N. Vanderbilt, R. Miesfeld, B. A. Maler, and K. R. Yamamoto, "Intracellular receptor concentration limits glucocorticoiddependent enhancer activity," Molecular Endocrinology, vol. 1, no. 1, pp. 68-74, 1987.

[10] C. Binbin, F. Yinglu, D. Juan, and L. Changquan, "Upregulation effect of ginsenosides on glucocorticoid receptor in rat liver," Hormone and Metabolic Research, vol. 41, no. 7, pp. 531-536, 2009.

[11] M. Li, C.-Q. Ling, X.-Q. Huang, and Z.-L. Shen, "Effects of ginsenosides extracted from ginseng stem and leaves on glucocorticoid receptor in different viscera in heat-damaged rats," Zhong Xi Yi Jie He Xue Bao, vol. 4, no. 2, pp. 156-159, 2006.

[12] C. Ling, Y. Li, X. Zhu, C. Zhang, and M. Li, "Ginsenosides may reverse the dexamethasone-induced down-regulation of glucocorticoid receptor," General and Comparative Endocrinology, vol. 140, no. 3, pp. 203-209, 2005.

[13] J. Li, J. Du, D. Liu et al., "Ginsenoside Rh1 potentiates dexamethasone's anti-inflammatory effects for chronic inflammatory disease by reversing dexamethasone-induced resistance," Arthritis Research and Therapy, vol. 16, no. 3, article R106, 2014.

[14] S. E. Wenderfer, B. Ke, T. J. Hollmann, R. A. Wetsel, H. Y. Lan, and M. C. Braun, "C5a receptor deficiency attenuates $\mathrm{T}$ cell function and renal disease in MRL ${ }^{I p r}$ mice," Journal of the American Society of Nephrology, vol. 16, no. 12, pp. 3572-3582, 2005.

[15] F. A. Kabeer, G. B. Sreedevi, M. S. Nair et al., "Antineoplastic effects of deoxyelephantopin, a sesquiterpene lactone from Ele- 
phantopus scaber, on lung adenocarcinoma (A549) cells," Journal of Chinese Integrative Medicine, vol. 11, no. 4, pp. 269-277, 2013.

[16] Y. Li, X. Wang, S. Cheng et al., "Diosgenin induces G2/M cell cycle arrest and apoptosis in human hepatocellular carcinoma cells," Oncology Reports, vol. 33, no. 2, pp. 693-698, 2014.

[17] B. Cheng, J. Li, J. Du, X. Lv, L. Weng, and C. Ling, "Ginsenoside $\mathrm{Rb} 1$ inhibits osteoclastogenesis by modulating NF- $\kappa \mathrm{B}$ and MAPKs pathways," Food and Chemical Toxicology, vol. 50, no. 5, pp. 1610-1615, 2012.

[18] X. Zhang, J. Zhu, W. Geng et al., "Electroacupuncture at Feishu (BL13) and Zusanli (ST36) down-regulates the expression of orexins and their receptors in rats with chronic obstructive pulmonary disease," Journal of Integrative Medicine, vol. 12, no. 5, pp. 417-424, 2014.

[19] T. J. Santoro, J. P. Portanova, and B. L. Kotzin, “The contribution of L3T4+ T cells to lymphoproliferation and autoantibody production in MRL-lpr/lpr mice," Journal of Experimental Medicine, vol. 167, no. 5, pp. 1713-1718, 1988.

[20] F. X. O’Sullivan, C. J. Ray, Y. Takeda, G. C. Sharp, and S. E. Walker, "Long-term anti-CD4 treatment of MRL/lpr mice ameliorates immunopathology and lymphoproliferation but fails to suppress rheumatoid factor production," Clinical Immunology and Immunopathology, vol. 61, no. 3, pp. 421-435, 1991.

[21] D. A. Jabs, C. L. Burek, Q. Hu, R. C. Kuppers, B. Lee, and R. A. Prendergast, "Anti-CD4 monoclonal antibody therapy suppresses autoimmune disease in MRL/Mp-lpr/lpr mice," Cellular Immunology, vol. 141, no. 2, pp. 496-507, 1992.

[22] H. Okazaki, D. Hirata, T. Kamimura et al., "Effects of FTY720 in MRL-lpr/lpr mice: therapeutic potential in systemic lupus erythematosus," Journal of Rheumatology, vol. 29, no. 4, pp. 707716, 2002.

[23] Y. Shi, S. Devadas, K. M. Greeneltch, D. Yin, R. A. Mufson, and J.-N. Zhou, "Stressed to death: Implication of lymphocyte apoptosis for psychoneuroimmunology," Brain, Behavior, and Immunity, vol. 17, supplement 1, pp. S18-S26, 2003.

[24] J. D. Amaral, S. Solá, C. J. Steer, and C. M. P. Rodrigues, "Role of nuclear steroid receptors in apoptosis," Current Medicinal Chemistry, vol. 16, no. 29, pp. 3886-3902, 2009.

[25] T. Dörner, C. Giesecke, and P. E. Lipsky, "Mechanisms of B cell autoimmunity in SLE," Arthritis Research \& Therapy, vol. 13, no. 5, article 243, 2011.

[26] H. Hanaoka, Y. Okazaki, T. Satoh et al., "Circulating antidouble-stranded DNA antibody-secreting cells in patients with systemic lupus erythematosus: a novel biomarker for disease activity," Lupus, vol. 21, no. 12, pp. 1284-1293, 2012.

[27] D. Villalta, N. Bizzaro, N. Bassi et al., "Anti-dsDNA antibody isotypes in systemic lupus erythematosus: $\operatorname{IgA}$ in addition to IgG anti-dsDNA help to identify glomerulonephritis and active disease," PLoS ONE, vol. 8, no. 8, Article ID e71458, 2013.

[28] H. Q. Qu, W. H. Bian, and Y. Y. Xu, "A novel NF- $\kappa$ B inhibitor, DHMEQ, ameliorates pristane-induced lupus in mice," Experimental and Therapeutic Medicine, vol. 8, no. 1, pp. 100-104, 2014.

[29] C. P. Nguyen, V. V. Cao, J. Fehér, and P. Gergely, "Correlation between the levels of antinuclear antibodies, anti-DNA antibodies, and complement in systemic lupus erythematosus.", Acta Medica Hungarica, vol. 45, no. 2, pp. 145-159, 1988.

[30] E. Paz, M. Adawi, I. Lavi, Y. Mussel, and R. Mader, "Antinuclear antibodies measured by enzyme immunoassay in patients with systemic lupus erythematosus: relation to disease activity," Rheumatology International, vol. 27, no. 10, pp. 941-945, 2007.
[31] A. Almogren, "Anti-double stranded antibody: association with titers and fluorescence patterns of anti-nuclear antibody in systemic lupus erythematosus," Saudi Medical Journal, vol. 31, no. 1, pp. 32-36, 2010.

[32] M. Herrmann, O. M. Zoller, M. Hagenhofer, R. Voll, and J. R. Kalden, "What triggers anti-dsDNA antibodies?" Molecular Biology Reports, vol. 23, no. 3-4, pp. 265-267, 1996.

[33] D.-R. Koh, A. Ho, A. Rahemtulla, W.-P. Fung-Leung, H. Griesser, and T.-W. Mak, "Murine lupus in MRL/lpr mice lacking CD4 or CD8 T cells," European Journal of Immunology, vol. 25, no. 9, pp. 2558-2562, 1995.

[34] D. A. Jabs, B. Lee, and R. A. Prendergast, "Role of T cells in the pathogenesis of autoimmune lacrimal gland disease in MRL/ Mp-lpr/lpr mice," Current Eye Research, vol. 16, no. 9, pp. 909916, 1997.

[35] L. Rönnblom and G. V. Alm, "A pivotal role for the natural interferon alpha-producing cells (plasmacytoid dendritic cells) in the pathogenesis of lupus," Journal of Experimental Medicine, vol. 194, no. 12, pp. F59-F63, 2001.

[36] B. M. Rüger, K. J. Erb, Y. He, J. M. Lane, P. F. Davis, and Q. Hasan, "Interleukin-4 transgenic mice develop glomerulosclerosis independent of immunoglobulin deposition," European Journal of Immunology, vol. 30, no. 9, pp. 2698-2703, 2000.

[37] A. Csiszár, G. Nagy, P. Gergely, T. Pozsonyi, and É. Pócsik, "Increased interferon-gamma (IFN- $\gamma$ ), IL-10 and decreased IL4 mRNA expression in peripheral blood mononuclear cells (PBMC) from patients with systemic lupus erythematosus (SLE)," Clinical and Experimental Immunology, vol. 122, no. 3, pp. 464-470, 2000.

[38] K. Sugimoto, S. Morimoto, H. Kaneko et al., "Decreased IL-4 producing CD4+ T cells in patients with active systemic lupus erythematosus-relation to IL-12R expression," Autoimmunity, vol. 35, no. 6, pp. 381-387, 2002.

[39] C. Y. Wu, C. Fargeas, T. Nakajima, and G. Delespesse, "Glucocorticoids suppress the production of interleukin 4 by human lymphocytes," European Journal of Immunology, vol. 21, no. 10, pp. 2645-2647, 1991.

[40] R. A. Daynes and B. A. Araneo, "Contrasting effects of glucocorticoids on the capacity of $\mathrm{T}$ cells to produce the growth factors interleukin 2 and interleukin 4," European Journal of Immunology, vol. 19, no. 12, pp. 2319-2325, 1989.

[41] H.-F. Xie, J. Li, and W. Shi, "Effect of corticosteroids on the balance of Th cytokines in patients with systemic lupus erythematosus," Hunan Yi Ke Da Xue Xue Bao, vol. 27, no. 6, pp. 533-535, 2002.

[42] D. K. L. Choy, F. Ko, S. T. Li et al., "Effects of theophylline, dexamethasone and salbutamol on cytokine gene expression in human peripheral blood CD4+ T-cells," European Respiratory Journal, vol. 14, no. 5, pp. 1106-1112, 1999.

[43] F. Ramírez, "Glucocorticoids induce a Th2 response in vitro," Developmental Immunology, vol. 6, no. 3-4, pp. 233-243, 1998.

[44] S. K. Agarwal and D. Marshall Jr., "Dexamethasone promotes type 2 cytokine production primarily through inhibition of type 1 cytokines," Journal of Interferon and Cytokine Research, vol. 21, no. 3, pp. 147-155, 2001. 


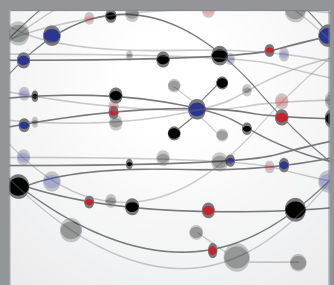

The Scientific World Journal
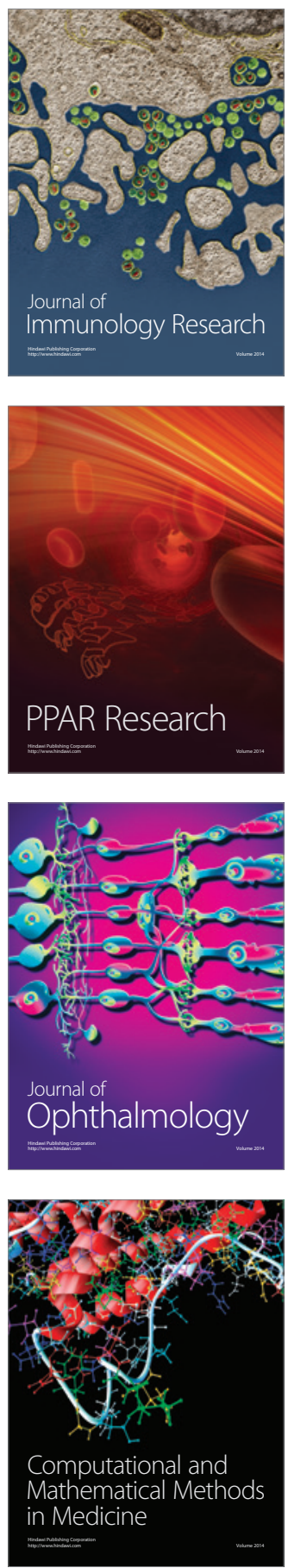

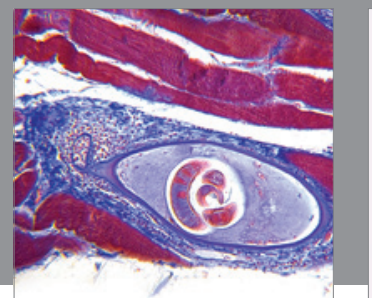

Gastroenterology

Research and Practice
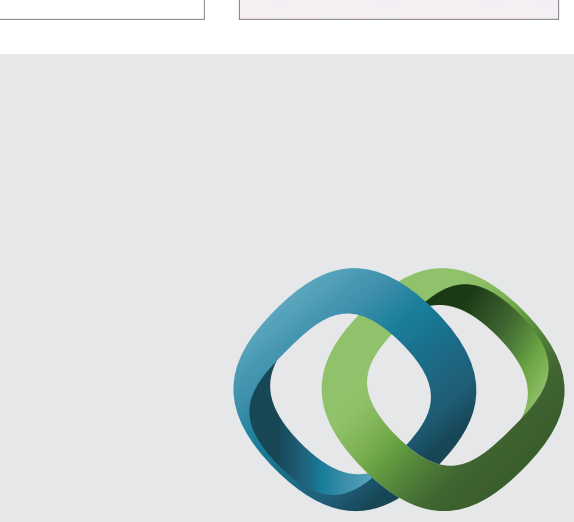

\section{Hindawi}

Submit your manuscripts at

http://www.hindawi.com
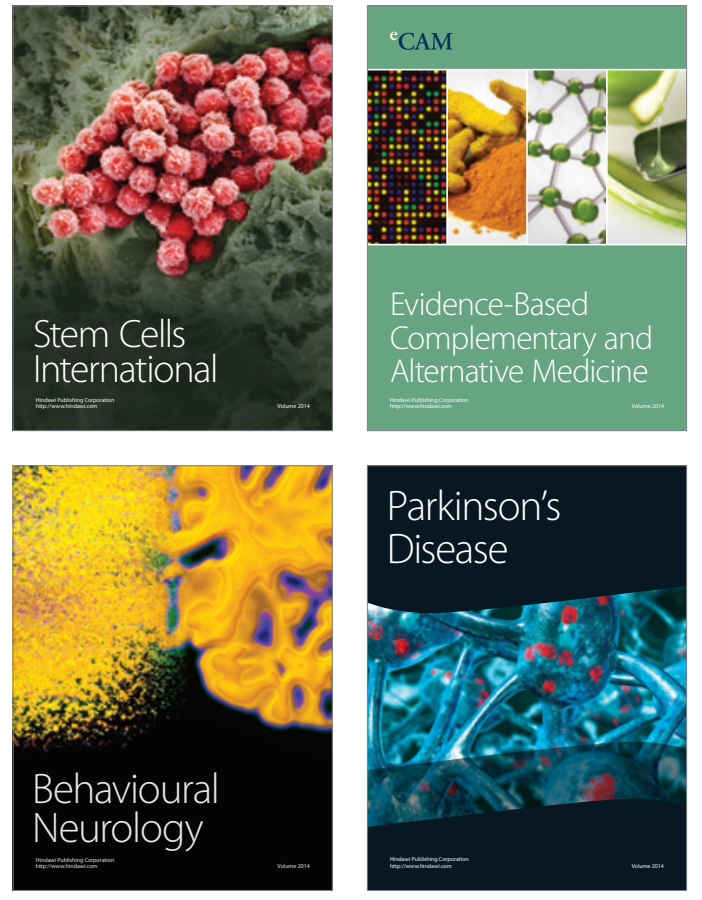
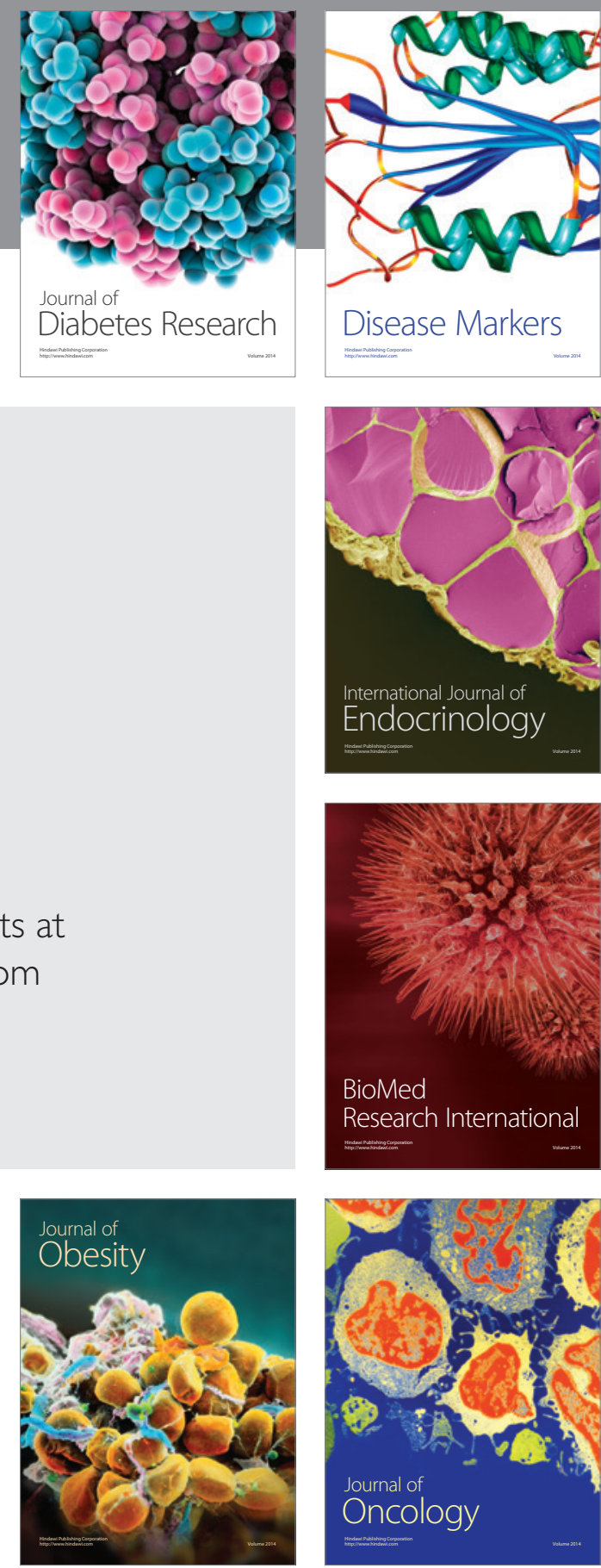

Disease Markers
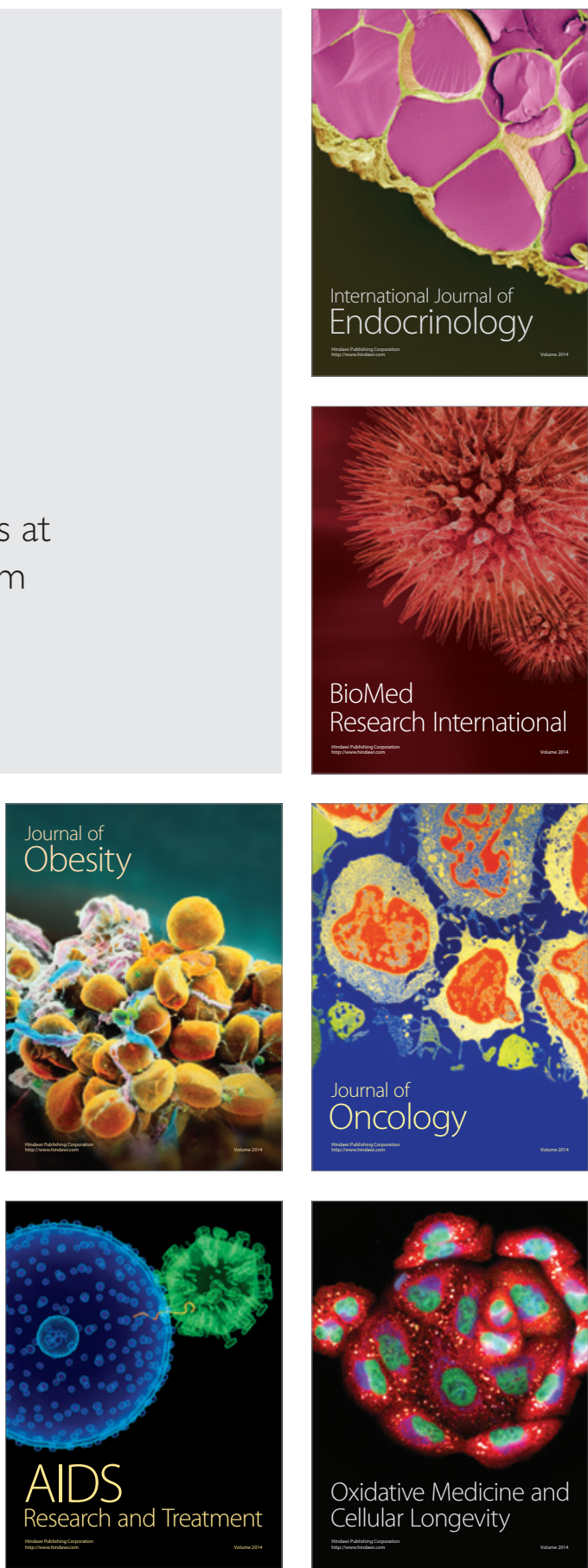